\title{
Análise das características físicas de comprimidos de losartana potássica 50mg dispensados em uma Unidade Básica de Saúde e em drogarias do município de
}

\author{
Abaetetuba-PA \\ Analysis of the physical characteristics of losartana potassium $50 \mathrm{mg}$ tablets dispensed in a Basic \\ Health Unit and in drugstores in the city of Abaetetuba-PA \\ Análisis de las características físicas de los tablets del losartán potásico 50mg dispensados en una \\ Unidad Básica de Salud y en farmacias del municipio de Abaetetuba-PA
}

Recebido: 09/11/2021 | Revisado: 18/11/2021 | Aceito: 22/11/2021 | Publicado: 28/11/2021

\author{
Maria Rosilda Valente de Sarges \\ ORCID: https://orcid.org/0000-0001-9689-718X \\ Universidade da Amazônia, Brasil \\ E-mail: mrvsfarma@gmail.com \\ Davi Araujo Souza \\ ORCID: https://orcid.org/0000-0001-8955-8411 \\ Universidade da Amazônia, Brasil \\ E-mail: daviaraujofarma@gmail.com \\ Vanessa Cavalcante Ribeiro \\ ORCID: https://orcid.org/0000-0002-9242-1982 \\ Universidade da Amazônia, Brasil \\ E-mail: vancavalcante29@gmail.com \\ Daniella Paternostro de Araújo Grisolia \\ ORCID: https://orcid.org/0000-0001-7204-4246 \\ Universidade da Amazônia, Brasil \\ E-mail: daniellapaternostro@gmail.com
}

\begin{abstract}
Resumo
A Hipertensão Arterial Sistêmica (HAS) é denominada uma condição clínica multifatorial, que ocorre quando os níveis pressóricos alcançam valores sustentáveis de $\geq 140$ sistólica e/ou 90 diastólica mmHg. Consequentemente, para tratamento e controle da doença, a losartana potássica $50 \mathrm{mg}$ é um anti-hipertensivo amplamente utilizado pela população devido ao baixo custo, boa efetividade e adesão ao tratamento. Diante disso, o presente artigo tem por objetivo identificar possíveis desvios de qualidade em comprimidos de losartana potássica 50mg, dispensados em uma unidade básica de saúde e em drogarias no município de Abaetetuba-PA. A fim de atingir o objetivo foram realizados testes de análise de identificação visual, peso médio e friabilidade, com a finalidade de comparar com a Farmacopeia Brasileira e identificar possíveis inconformidades. Os resultados obtidos na análise visual foram parâmetros normais de cor, odor e integridade dos comprimidos e embalagens. No teste de peso médio os resultados foram variações de $1,32 \%$ nos comprimidos similares, $1,64 \%$ nos comprimidos genéricos e 1,02\% nos comprimidos de referência. No teste de friabilidade o máximo de perda atingida foi os comprimidos similares com $0,13 \%$, e o mínimo foram os comprimidos de referência com $0,1 \%$. Dessa forma, os resultados demonstraram perfil compatível com o estabelecido na Farmacopeia Brasileira, tendo percentuais de variação dentro do limite instituído. Deste modo, conclui-se que os comprimidos de losartana potássica 50mg dispensados no município possuem qualidade, portanto, garantindo o efetivo tratamento de seus pacientes acometidos pela HAS.
\end{abstract}

Palavras-chave: Análises; Losartana potássica; Comprimidos; Características físicas.

\begin{abstract}
Systemic Arterial Hypertension (SAH) is a multifactorial clinical condition characterized by sustained elevation of blood pressure levels $\geq 140$ systolic and/or 90 diastolic mmHg. Consequently, for treatment and control of the disease, losartan potassium $50 \mathrm{mg}$ is an antihypertensive widely used by the population. Therefore, the present article aims to identify possible quality deviations in losartana potassium $50 \mathrm{mg}$ tablets dispensed in a basic health unit and drugstores in the city of Abaetetuba-PA. In order to achieve the objective, analysis visual identification, average weight and friability were performed in order to compare with the Brazilian Pharmacopoeia and identify possible non-conformities. The results obtained in the visual analysis were normal parameters of color, odor and integrity of the tablets and packaging. In the average weight test the results were variations of $1.32 \%$ in similar pills, $1.64 \%$ in generic pills, and $1.02 \%$ in reference pills. In the friability test the maximum loss reached was the similar tablets with $0.13 \%$, and the
\end{abstract}


minimum was the reference tablets with $0.1 \%$. Thus, the results showed a profile compatible with that established in the Brazilian Pharmacopoeia, with variation percentages within the established limit. Thus, it is concluded that losartana potassium 50mg tablets dispensed in the city have quality, thus ensuring the effective treatment of patients suffering from SAH.

Keywords: Analysis; Losartan potassium; Tablets; Physical characteristics.

\section{Resumen}

La hipertensión arterial sistémica (HSA) es una condición clínica multifactorial caracterizada por la elevación sostenida de los niveles de presión arterial $\geq 140$ sistólica y/o 90 diastólica $\mathrm{mmHg}$. En consecuencia, para el tratamiento y control de la enfermedad, losartana potasio $50 \mathrm{mg}$ es un antihipertensivo ampliamente utilizado por la población. El presente artículo tiene como objetivo identificar posibles desviaciones de calidad en tabletas de losartán potásico 50mg, dispensados en una unidad básica de salud y en farmacias del municipio de Abaetetuba-PA. Para alcanzar el objetivo, se realizaron análisis de identificación visual, peso medio y friabilidad para comparar con la Farmacopea Brasileña e identificar posibles inconsistencias. Los resultados obtenidos en el análisis visual fueron los parámetros normales de color, olor e integridad de los comprimidos y del envase. En la prueba del peso medio, los resultados fueron variaciones del $1,32 \%$ en los comprimidos similares, del 1,64\% en los comprimidos genéricos y del 1,02\% en los comprimidos de referencia. En la prueba de friabilidad la pérdida máxima alcanzada fue la de los comprimidos similares con un $0,13 \%$, y la mínima la de los comprimidos de referencia con un $0,1 \%$. Así, los resultados demostraron un perfil compatible con el establecido en la Farmacopea Brasileña, con porcentajes de variación dentro del límite establecido. Así pues, se concluyó que los comprimidos de losartana potásica de $50 \mathrm{mg}$ dispensados en la ciudad son de calidad, lo que garantiza el tratamiento eficaz de los pacientes que padecen HSA.

Palabras clave: Análisis; Losartán potásico; Tabletas; Características físicas.

\section{Introdução}

A Organização Mundial da Saúde (OMS) (1994) define estabilidade farmacêutica como a capacidade do produto farmacêutico manter a suas propriedades químicas, físicas, microbiológicas e biofarmacêuticas dentro dos limites especificados durante todo o seu prazo de validade. Nesse sentido, a análise de qualidade física visa verificar a qualidade e integridade do medicamento mantendo estabilidade e segurança (Shantier, 2020). Os medicamentos devem ser produzidos de acordo com a farmacopeia brasileira e devem passar por teste de conformidade em sua fabricação, antes de serem comercializados. Os ensaios em série são indispensáveis para aferir a conformidade desse medicamento, com as especificações físico-químicas e microbiológicas, para estipular estabilidade e qualidade do produto (Kopp, 2006).

O mercado brasileiro de medicamentos, era, até então, constituído por duas categorias de medicamentos: os “inovadores", atualmente designados como medicamentos de referência, que além de marca própria, são registrados mediante garantia comprovada de eficácia e segurança; e os "similares", que são compostos com o mesmo ou os mesmos princípios ativos, a mesma concentração, forma farmacêutica, via de administração, posologia e indicação terapêutica do medicamento de referência registrado na Anvisa (Brasil, 2001). A partir de 1999, uma nova classe, os medicamentos genéricos, adentrou no mercado farmacêutico nacional. Tais medicamentos, além de possuírem as mesmas propriedades dos similares, devem evidenciar que são intercambiáveis com os medicamentos de referência registrados na Anvisa. Assim, devem apresentar resultados que garantam equivalência farmacêutica e, se for o caso, biodisponibilidade relativa (ou biodisponibilidade) ao produto de referência (Bertoldi et al., 2016). Logo, a importância de garantir a qualidade e estabilidade desses medicamentos amplamente utilizados na população é primordial, pois é necessário que o medicamento, no momento do uso, tenha ação farmacológica preservada e perfil toxicológico seguro (Lombardo \& Eserian, 2017). A estabilidade pode ser afetada pelos meios de conservação e acondicionamentos aplicados, como exposição a umidade, temperatura e luminosidade, dessa forma interferindo diretamente no fármaco ou na formulação em geral (Shantier, 2020).

A Hipertensão Arterial Sistêmica (HAS) é uma condição clínica multifatorial caracterizada por elevação sustentada dos níveis pressóricos $\geq 140$ sistólica e/ou 90 diastólica $\mathrm{mmHg}$, geralmente associados a certos distúrbios metabólicos e alterações funcionais, que podem ser potencializados por outros fatores de risco, como dislipidemia, obesidade abdominal, intolerância à glicose e Diabetes mellitus (Barroso et al., 2021). Esta patologia é, atualmente, considerada, umas das doenças que mais afeta a 
população do planeta, e no Brasil, atinge cerca de 32,5\% da população adulta (Malachias et al., 2016). Um dos mecanismos para o controle da HAS é por meio do bloqueio dos receptores de angiotensina I (AT1) diminuindo a ativação desses receptores pela angiotensina II. Seus efeitos farmacológicos são similares aos dos inibidores da enzima conversora de angiotensina (ECA) por produzirem dilatação arteriolar e venosa e bloqueio da secreção de aldosterona, reduzindo, assim, a pressão arterial e diminuindo a retenção de sal e água (Frota, 2019).

Dos medicamentos para tratamento da HAS que estão presentes na Relação Nacional de Medicamentos Essenciais (RENAME) (2020), no Sistema Único de Saúde (SUS), encontra-se o comprimido de losartana potássica $50 \mathrm{mg}$, fármaco da classe dos antagonistas do receptor de angiotensina II, amplamente utilizado pela população (Al-Majed et al., 2015).

$\mathrm{Na}$ literatura científica existem vários trabalhos avaliando a qualidade das características físicas dos medicamentos com objetivo de disponibilizar um produto de qualidade, seguro e eficaz para os usurários. Diante disso, várias pesquisas têm sido realizadas como a determinação de peso médio, desintegração e teor de cápsulas de hidroclorotiazida manipuladas, as quais mostram que há valores insuficientes de doseamento esperado (Aglio, 2016). Outro estudo demonstrou que as pesquisas e análises brasileiras, envolvidas ao controle de qualidade de medicamentos, indicam resultados que variam dentro dos valores estabelecidos na farmacopeia brasileira e internacionais, sendo que esses valores podem significar instabilidade na segurança dos medicamentos (Barata-Silva, Hauser-Davis, Silva \& Moreira, 2017). Em 2014, Royo, Araújo e Saldanha, Xavier, Freitas e Menezes analisaram o peso médio e a desintegração do losartana potássica manipulado em farmácias em Minas Gerais, os dados encontrados estavam de acordo com farmacopeia.

Visando um olhar mais específico para os munícipios do interior da região norte, esta pesquisa foca-se no município de Abaetetuba, localizado no interior do Pará, na Amazônia Brasileira, é caracterizado pelo clima tropical quente e úmido. Esta caracterização climática de Abaetetuba é um dos fatores que podem interferir na qualidade dos medicamentos (Lima, Costa \& Santos-Fonseca, 2020). Considerando que o transporte, armazenamento e distribuição deste medicamento quando feitos de forma ineficaz, de modo a comprometer a qualidade e segurança, pode colocar em risco a vida do paciente e agravar seu quadro clínico. Nesse sentido, o estudo objetiva investigar possíveis desvios de qualidade através de análises físicas de comprimidos de losartana potássica 50mg similares disponíveis em uma unidade básica de saúde, e possíveis não conformidades entre comprimidos de medicamentos de referência e genérico dispensados em drogarias do município.

\section{Metodologia}

\subsection{Obtenção das amostras}

As amostras foram adquiridas em drogarias do município de Abaetetuba-PA. O município possui aproximadamente $1.610,743 \mathrm{~km}^{2}$, sua população é chamada de abaetetubenses, e o mesmo contava com cerca de 141.100 habitantes no último censo realizado em 2010 de acordo com o Instituto Brasileiro de Geografia e Estatística (IBGE) (2020). Estima-se a quantidade de 159.080 habitantes até 2020 segundo o IBGE (2020). As amostras utilizadas foram os comprimidos de losartana potássica 50mg obtidas na unidade básica de saúde, denominada "Centro de Saúde Dr. Heraldo Pantoja" e em duas drogarias do mesmo município. A unidade básica de saúde e as drogarias foram escolhidas aleatoriamente entre os locais de mais movimento e atendimento de pacientes. Os comprimidos concedentes pela unidade básica de saúde foram similares, já os comprimidos obtidos a partir de drogarias foram genéricos e de referência. Os testes foram realizados no Laboratório de Ciências Farmacêuticas do campus Alcindo Cacela, na Universidade da Amazônia. Para a realização dos testes foram utilizados 20 comprimidos em cada teste das amostras (genérico, similar e referência). A Tabela 1 exibe informações sobre as amostras adotadas para o estudo. 
Tabela 1 - Informações dos comprimidos utilizados na pesquisa.

\begin{tabular}{llll}
\hline & Similar & Genérico & Referência \\
\hline Lote & 2106291 & 21011084 & V006179 \\
\hline Data de validade & $05 / 2023$ & $02 / 2023$ & $11 / 2023$ \\
\hline
\end{tabular}

Fonte: Autores (2021).

\subsection{Aspecto visual}

Foi analisado o aspecto visual das amostras quanto a uniformidade de coloração dos comprimidos, comprimidos faltantes, quebrados ou trincados ou qualquer outra alteração aparente. A Farmacopeia Brasileira caracteriza fisicamente a losartana potássica como pó cristalino branco ou quase branco (Brasil, 2019; Guimarães, 2018).

\subsection{Peso médio}

O teste de peso médio foi realizado através da média aritmética obtida na pesagem individual de 20 unidades, que foram retirados aleatoriamente de uma mesma embalagem, em balança analítica AG200 previamente calibrada, conseguinte, a partir do peso médio determinou-se variação do peso entre os comprimidos através do desvio padrão relativo, de acordo com o estabelecido pela Farmacopeia Brasileira (Brasil, 2019). Para os pesos obtidos foi utilizado o programa Microsoft Excel 2019 no armazenamento de dados, cálculos e na criação de tabelas e gráficos.

\subsection{Friabilidade}

No teste de friabilidade, foi realizada a pesagem de 20 comprimidos da mesma embalagem em uma balança analítica AG200 previamente calibrada. Os comprimidos foram levados ao friabilômetro MOD.300, durante 5 minutos numa velocidade de rotação de 25 rotações por minuto. Após 5 minutos, apenas comprimidos íntegros e sem resíduos foram retirados do aparelho, e, novamente pesados. Foi calculada a perda percentual de massa de 20 comprimidos baseada na diferença do peso inicial e peso final que de acordo com as especificações farmacopeicas que estabelece que os valores sejam iguais ou inferiores a 1,5\% (Brasil, 2019). A Figura 2 representa o aparelho friabilômetro.

Figura 1 - Aparelho para teste de friabilidade (friabilômetro).

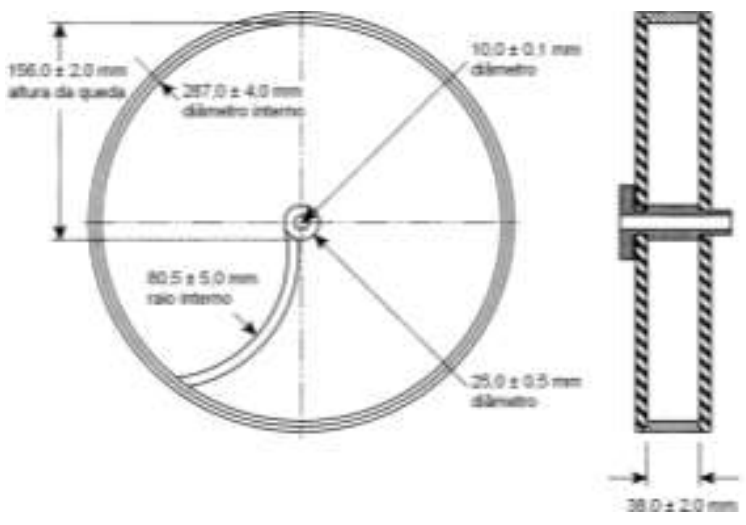

Fonte: Brasil (2019).

Na Figura 2 são expostas especificações obrigatórias que um friabilômetro deve possuir quanto ao formato e tamanho do equipamento. Os resultados foram organizados em planilhas do Microsoft Excel 2019 e analisados a partir de tabelas e gráficos. 


\section{Resultados e Discussão}

\subsection{Características visuais}

Os três lotes de medicamentos similar, genérico e referência estavam dentro dos parâmetros normais de cor cristalino branco, odor característico, integridade dos comprimidos, embalagens primárias e secundárias íntegras e informações necessárias nas embalagens conforme a Farmacopeia Brasileira (Brasil, 2019). As características visuais de uniformidade de coloração dos comprimidos, comprimidos faltantes, quebrados ou trincados, legibilidade na embalagem e qualquer outra alteração aparente corroboram com os dados de Bianchin et al. (2012), que avaliaram a qualidade de outros medicamentos anti-hipertensivos distribuídos na rede pública de saúde de um município do estado de Santa Catarina e obtiveram dados semelhantes a este estudo.

\subsection{Determinação de peso médio}

O peso médio consiste em avaliar os comprimidos de um mesmo lote quanto a uniformidade de peso (Brasil, 2019). No presente estudo foi utilizado lotes de medicamentos similar, genérico e referência. Os resultados do peso individual e as médias são visíveis na Tabela 2.

Tabela 2 - Determinação do peso médio de comprimidos de losartana potássica 50 mg.

\begin{tabular}{|c|c|c|c|}
\hline Amostras & $\begin{array}{l}\text { Similar } \\
\text { Peso (mg) }\end{array}$ & $\begin{array}{l}\text { Genérico } \\
\text { Peso (mg) }\end{array}$ & $\begin{array}{l}\text { Referência } \\
\text { Peso (mg) }\end{array}$ \\
\hline 1 & 158,4 & 165,3 & 155,7 \\
\hline 2 & 158,6 & 158,3 & 154,1 \\
\hline 3 & 158,4 & 158,8 & 157,5 \\
\hline 4 & 157,3 & 157,3 & 156,3 \\
\hline 5 & 155,6 & 164,4 & 157,1 \\
\hline 6 & 155,9 & 161,8 & 152,6 \\
\hline 7 & 158 & 161,7 & 157,1 \\
\hline 8 & 159,9 & 163,6 & 152,6 \\
\hline 9 & 154,7 & 161,3 & 154,4 \\
\hline 10 & 156,3 & 158,7 & 158,3 \\
\hline 11 & 160 & 163,7 & 155,7 \\
\hline 12 & 155 & 161,1 & 154,5 \\
\hline 13 & 156,9 & 158 & 155,1 \\
\hline 14 & 153,7 & 160,6 & 156,3 \\
\hline 15 & 155,5 & 157 & 156,1 \\
\hline 16 & 159 & 157,2 & 155,1 \\
\hline 17 & 157,7 & 160,3 & 156,7 \\
\hline 18 & 152,2 & 162,5 & 153,7 \\
\hline 19 & 155 & 157,4 & 157,1 \\
\hline 20 & 157,2 & 158,1 & 154,9 \\
\hline Peso Médio & 156,765 & 160,355 & 155,545 \\
\hline Desvio Padrão Relativo & $1,32 \%$ & $1,64 \%$ & $1,02 \%$ \\
\hline
\end{tabular}

Fonte: Autores (2021).

De acordo com os resultados expostos na Tabela 2, o peso médio obtido do medicamento similar corresponde a 156,765 mg, genérico 160,355 mg e referência 155,545 mg. A Farmacopeia Brasileira estabelece limite de variação de máximo 7,5\% para comprimidos com mais de $80 \mathrm{mg}$ e menos de $250 \mathrm{mg}$ no peso médio, como a losartana potássica (Brasil, 2019). As variações 
em desvio padrão relativo foram de 1,32\% nos comprimidos similares, 1,64\% nos comprimidos genéricos e $1,02 \%$ nos comprimidos de referência. Estes dados são similares ao estudo de Carvalho, Lira, Sabino, Barros, Mendonça, Sousa e Medeiros (2019), que avaliou o peso médio do medicamento losartana potássica mais hidroclorotiazida nas apresentações de referência e genérico, no município de Sobral- CE, em que o menor peso foi de 306,9 mg e o maior peso obtido foi de 314,8 mg, de modo que todos os medicamentos estavam dentro do limite de variação de peso dos comprimidos. O Gráfico 1 indica a variação do desvio padrão relativo entre os medicamentos.

Gráfico 1 - Variação do desvio padrão relativo.

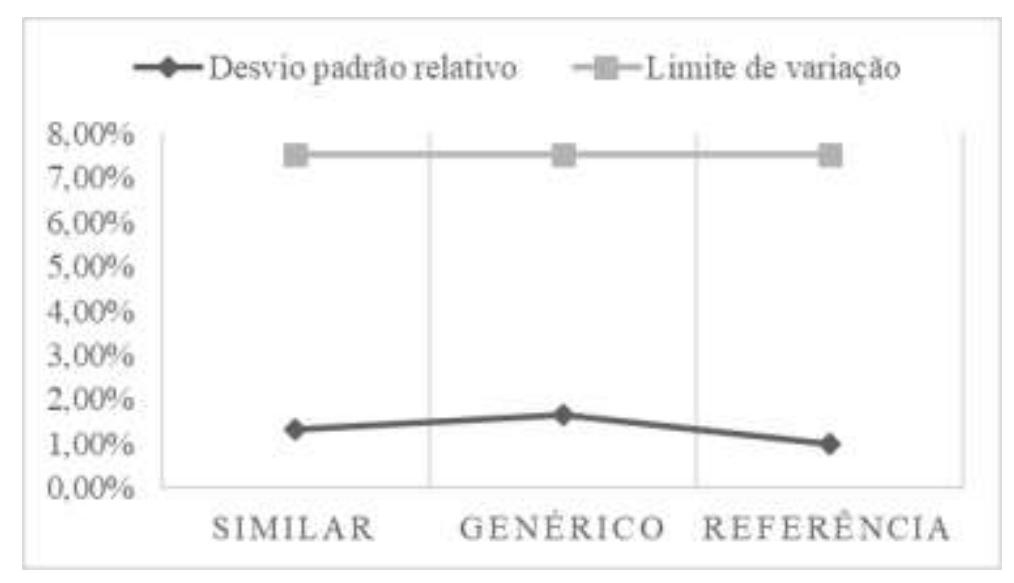

Fonte: Autores (2021).

De acordo com o Gráfico 1, todos os três lotes obedeceram aos limites de variação empregados na Farmacopeia Brasileira (Brasil, 2019). Os medicamentos similar, genérico e referência não ultrapassaram $2 \%$ de desvio padrão relativo. Nesse sentido, todos os comprimidos analisados correspondiam à regularidade no peso médio por lote.

Este resultado está de acordo com Andrade, Santos e Siqueira (2019) no qual avaliaram a determinação do peso médio de medicamentos obtidos em drogarias atendentes do sistema Farmácia Popular do Brasil, na cidade de Caruaru-PE, em que também nenhum comprimido se apresentou acima das variações em porcentagens.

\subsection{Teste de friabilidade}

A friabilidade é um teste que permite definir a resistência dos comprimidos a desgastes como rachaduras e perda de pó com o princípio ativo. O teste necessita do aparelho friabilômetro para simular ações mecânicas que o comprimido pode sofrer em condições de transporte. A friabilidade é exposta pela diferença entre o peso inicial e o final, representada em forma de porcentagem de pó perdido como no estudo de Andrade et al. (2019). O Gráfico 2 aborda os resultados do peso em gramas antes e após o teste no friabilômetro. 
Gráfico 2 - Resultados do teste de friabilidade.

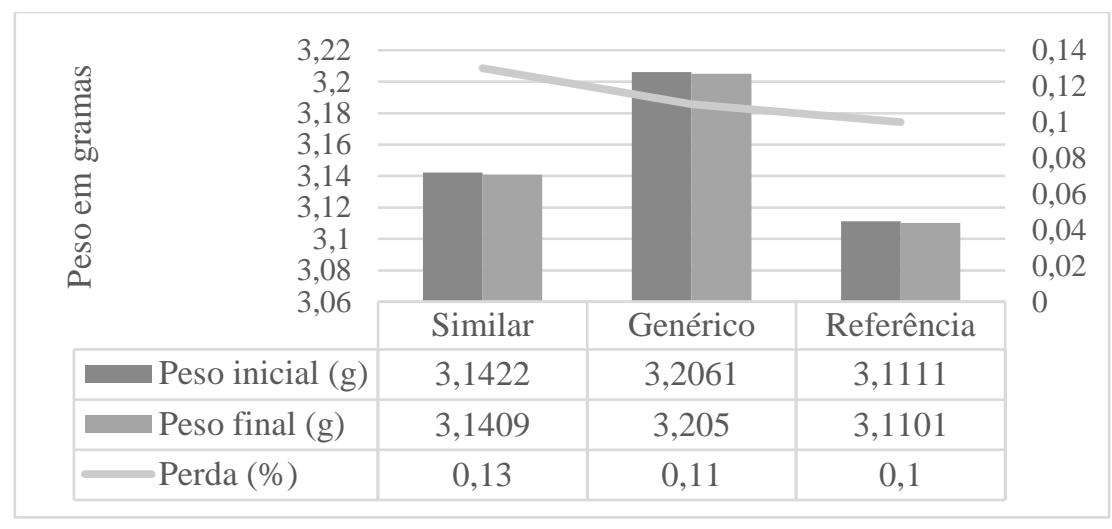

Fonte: Autores (2021).

O máximo de perda atingida foi os comprimidos similares com $0,13 \%$, e o mínimo foram os comprimidos de referência com $0,1 \%$ de perda, sendo aceitáveis os comprimidos com perda igual ou inferior a 1,5\% do seu peso descrito na Farmacopeia Brasileira (Brasil, 2019). Os resultados para o teste de friabilidade foram satisfatórios. Conforme o Gráfico 2, todos os lotes, de medicamento similar, genérico e referência, tiveram perda aceitável. Não houve, após o teste, comprimidos quebrados, lascados, rachados ou partidos. O estudo de Carvalho et al. (2019) apresentou resultados semelhantes na avaliação de friabilidade do medicamento losartana potássica mais hidroclorotiazida, nas apresentações de referência e genérico, no município de SobralCE, no qual o medicamento de referência não teve variação e o genérico a variação foi de $0,01 \%$ e os comprimidos mantiveramse íntegros após o teste.

\section{Considerações Finais}

O estudo demonstrou que os comprimidos analisados condizem aos parâmetros expostos pela Farmacopeia Brasileira (Brasil, 2019). Dessa forma, podemos inferir que os comprimidos de losartana potássica $50 \mathrm{mg}$, dispensados tanto na UBS parceira do projeto quanto nas drogarias no município de Abaetetuba - PA, são adquiridos, transportados e armazenados de forma adequada. Não houve diferenciais de dados discrepantes entre o medicamento similar dispensado na UBS e os medicamentos genérico e de referência dispensados pelas drogarias. Os comprimidos estão dentro da qualidade pelos testes realizados, nesse contexto oferecendo medicamento de qualidade e beneficiando a população do município. Todavia, dentro da literatura científica, há poucos estudos de análises em comprimidos de losartana potássica, de forma que não existem muitos dados comparativos. Desta forma, mais estudos nesta linha de pesquisa precisam ser realizados.

\section{Agradecimentos}

Ao Centro de Saúde Dr. Heraldo Pantoja por ceder espaço, atenção e as amostras de medicamentos similares para esta pesquisa. À Universidade da Amazônia por ofertar estrutura no laboratório. À professora Dra. Daniella Paternostro de Araújo pela orientação e apoio técnico.

\section{Referências}

Aglio, T. C. Determinação de Peso Médio, Desintegração e Teor de Cápsulas de Hidroclorotiazida Manipuladas em Farmácias Magistrais de Juiz de Fora MG. 2016. 47f. Trabalho de conclusão de curso (TCC) em graduação em Farmácia. Faculdade de Farmácia da Universidade Federal de Juiz de Fora, 2016. 
Andrade, E. G., Santos, C. O \& Siqueira, L. P. (2019). Controle físico-químicos de losartana potássica dispensado no programa Farmácia Popular do Brasil na cidade de Caruaru-PE. In M. C. Silva (Coord.), Políticas de saúde para o envelhecimento populacional (pp. 153-163). Ponta Grossa: Atena Editora. https://doi.org/10.22533/at.ed.57119191216

Barata-Silva, C., Hauser-Davis, R. A., Silva, A. L. O. \& Moreira, J. C. (2017). Desafios ao controle da qualidade de medicamentos no Brasil. Caderno de Saúde Coletiva, 25(3), 362-370. https://doi.org/10.1590/1414-462X201700030075

Barroso, W. K. S., Rodrigues, C. I. S., Bortolotto, L. A., Mota-Gomes, M. A., Brandão, A. A., Feitosa, A. D. M. et al. (2021). Diretrizes Brasileiras de Hipertensão Arterial- 2020. Arquivos Brasileiros de Cardiologia, 116(3), 516-658. https://doi.org/10.36660/abc.20201238

Bertoldi, A. D., Arrais, P. S. D., Tavares, N. U. L., Ramos, L. R., Luiza, V. L., Mengue, S. S., Dal-Pizzol, T. S., Farias, M. R. \& Oliveira, M. A. (2016). Utilização de medicamentos genéricos na população brasileira: uma avaliação da PNAUM 2014. Revista de Saúde Pública, 50(2), 11. https://doi.org/10.1590/S15188787.2016050006120

Brasil. (2001). Decreto $n^{\circ} 3.961$ de 10.10.2001: Altera o Decreto ${ }^{\circ} 79.094$, de 5 de janeiro de 1997, que regulamenta a Lei ${ }^{\circ} 6.360$, de 23 de setembro de 1976 . Diário Oficial União. Brasília.

Brasil. (2019). Agência Nacional de Vigilância Sanitária. Farmacopeia Brasileira. (6aed). Anvisa, v.1.

Brasil. (2019). Agência Nacional de Vigilância Sanitária. Farmacopeia Brasileira. (6aed). Anvisa, v.2.

Bianchin, M. D., Blatt, C. R., Soares, A. S. \& Külkamp-Guerreiro, I. C. (2012). Avaliação da qualidade de comprimidos de propranolol e enalapril distribuídos no sistema público de saúde em uma cidade do sul do Brasil. Ciência \& Saúde Coletiva, 17(2):491-498. https://doi.org/10.1590/S1413-81232012000200022

Carvalho, T. V., Lira, J. V., Sabino, A. P., Barros, A. E. V., Mendonça, A. C. S., Sousa, I. L. L. \& Medeiros, D. P. F. (2019). Avaliação da resistência mecânica do medicamento de referência e genérico: losartana potássica + hidroclorotiazida. In B. R. Silva Neto (Coord), Saúde Pública e Saúde Coletiva: Dialogando sobre Interfaces Temáticas 5 (pp. 55-62). Ponta Grossa, PR: Atena Editora. https://doi.org/10.22533/at.ed.9901902095

Frota, V. H. Avaliação quantitativa de losartana potássica (50 mg) em comprimidos de referência, genéricos e similares dispensados nas drogarias do Distrito Federal. 2019. 15f. Trabalho de conclusão de curso (TCC) em graduação em Farmácia. Centro Universitário do Planalto Central Aparecido dos Santos, 2019.

Guimarães, S. Estudo do teor, impurezas e polimorfismo da losartana no insumo farmacêutico ativo e no produto acabado. 2018.174 f. Dissertação (de mestrado), Instituto Nacional de Controle de Qualidade em Saúde, Fundação Oswaldo Cruz, Rio de Janeiro, 2018.

Instituto Brasileiro de Geografia e Estatística. (2017). Panorama do município de Abaetetuba: População no último censo 2010. Recuperado de https://cidades.ibge.gov.br/brasil/pa/abaetetuba/panorama. Acesso em 20 de abril de 2021.

Lima, F. B., Costa, J. M. \& Santos-Fonseca, D. J. (2020). Medicinal plants used in the public health system of Abaetetuba, in the Brazilian Amazon. GSC Biological and Pharmaceutical Sciences, 12(1): 80-86. https://doi.org/10.30574/gscbps.2020.12.1.0178

Kopp, S. (2006). Stability testing of pharmaceutical products in a global environment. Regulatory Affairs Journal Pharma. Informa UK, 16, 291-294. https://www.researchgate.net/profile/Sabine-Kopp-2/publication/284463697_Stability_testing_of_pharmaceutical_products_in_a_global_environment/links/ 5fb4055 b299b f10c36874e3b/Stability-testing-of-pharmaceutical-products-in-a-global-environment.pdf

Lombardo, M. \& Eserian, J. K. (2017). A análise da qualidade de medicamentos e o papel do laboratório oficial no contexto da saúde pública. Revista de Administração em Saúde, 17(67), 23. http://dx.doi.org/10.23973/ras.67.28

Malachias, M. V. B., Souza, W. K. S. B., Plavnik, F. L., Rodrigues, C. I. S., Brandão, A. A. \& Neves, M. F. T. et al (2016). $7^{\text {a }}$ Diretriz Brasileira de Hipertensão Arterial. Arquivos Brasileiros de Cardiologia, 107(3): 25-28. https://doi.org/10.5935/abc.20160155

Organização Mundial da Saúde. (1994). Pharmaceuticals Unit OMS guidelines on stability testing of pharmaceutical products containing well-established drug substances in conventional dosage forms. World Health Organization. https://apps.who.int/iris/handle/10665/62169

Relação Nacional de Medicamentos Essenciais (2020). Ministério da Saúde, Secretaria de Ciência, Tecnologia, Inovação e Insumos Estratégicos em Saúde, Departamento de Assistência Farmacêutica e Insumos Estratégicos. Brasília, Ministério da Saúde. http://conitec.gov.br/images/Rename-2020-final.pdf

Royo, V. A., Araújo e Saldanha, K. L., Xavier, M. P., Freitas, R. F. \& Menezes, E. V. (2014). Análise do medicamento losartana potássica manipulado em farmácias de Montes Claros, MG. Infarma, 26(4), 229-232. http://dx.doi.org/10.14450/2318-9312.v26.e4.a2014.pp229-232

Shantier, S. (2020). Drug Analysis. In Ahmad, U. \& Akhtar, J. Drug Analysis Pharmaceutical Formulation Design Recent Practices. (pp. 23-40). London: Intech Open. http://dx.doi.org/10.5772/intechopen.88739 